\title{
Perturbative QCD relations inspired by hypothetical tau leptons *
}

\author{
J.R.Peláez ${ }^{\mathrm{a}}$, S.J.Brodsky ${ }^{\mathrm{b}}$, C.Merino ${ }^{\mathrm{c}}$ and N. Toumbas ${ }^{\mathrm{d}}$ \\ ${ }^{a}$ Departamento de Física Teórica. Universidad Complutense de Madrid. \\ 28040 Madrid. SPAIN \\ ${ }^{\mathrm{b}}$ Stanford Linear Accelerator Center. Stanford University. \\ Stanford, California 94309. U.S.A. \\ ${ }^{\mathrm{c}}$ Departamento de Física de Partículas. Universidade de Santiago de Compostela. \\ 15706 Santiago de Compostela. SPAIN
}

${ }^{\mathrm{d} S t a n f o r d}$ Linear Accelerator Center. Stanford University. Stanford, California 94309. U.S.A.

\begin{abstract}
We review our recent works on tests of perturbative QCD, inspired by the relation between the hadronic decay of the $\tau$ lepton and the $e^{+} e^{-}$annihilation into hadrons. First, we present a set of commensurate scale relations that probe the self-consistency of leading-twist QCD predictions for any observable which defines an effective charge. These tests are independent of the renormalization scheme and scale, and are applicable over wide data ranges. As an example we apply this approach to $R_{e^{+} e^{-}}$. Second, using a differential form of these conmensurate scale relations, we present a method to measure the QCD Gell-Mann-Low $\Psi$ function.
\end{abstract}

\section{Introduction}

The hadronic width of the $\tau$ lepton, $R_{\tau}=$ $\Gamma\left(\tau^{-} \rightarrow \nu_{\tau}+\right.$ hadrons $) / \Gamma\left(\tau^{-} \rightarrow \nu_{\tau} e^{-} \overline{\nu_{e}}\right)$ plays an important role in the determination of the QCD coupling [1]. For this analysis it is essential to minimize the sensitivity to low energy data [2] using different integral moments. In addition, by integrating the data on spectral functions up $M$ we can simulate the physics of hypothetical $\tau$ leptons 2] with smaller $M$ than the real one. Since their hadronic widths are related to the $e^{+} e^{-}$annihilation cross section into hadrons $R_{e^{+} e^{-}}$through

$$
\begin{aligned}
& R_{\tau}(M)=\frac{2}{\left(\sum_{f} q_{f}^{2}\right)} \times \\
& \int_{0}^{M^{2}} \frac{d s}{M^{2}}\left(1-\frac{s}{M^{2}}\right)^{2}\left(1+\frac{2 s}{M^{2}}\right) R_{e^{+} e^{-}}(\sqrt{s}) .
\end{aligned}
$$

they provide tests of perturbative QCD (PQCD).

Our aim in this paper is to report on several

\footnotetext{
${ }^{*}$ Research partially supported by the Spanish CICYT under contract AEN97-1693 and the U.S. Department of Energy DE-AC03-76SF00515.
}

different applications motivated by the above relation, where we proposed self-consistency tests of PQCD, applicable to any observable which defines an effective charge [3], as well as a method to measure the Gell-Mann-Low function of some of these observables [1].

An effective charge defined from an observable contain its entire radiative contribution [6]. For instance, assuming $f$ massless flavors, we have

$$
\begin{aligned}
R_{e^{+} e^{-}}(\sqrt{s}) & \equiv R_{0}\left[1+\frac{\alpha_{R}(\sqrt{s})}{\pi}\right], \\
R_{\tau}(M) & \equiv R_{\tau}^{0}(M)\left[1+\frac{\alpha_{\tau}(M)}{\pi}\right],
\end{aligned}
$$

where $R_{0}=3 \sum_{f} q_{f}^{2}$ The effective charges $\alpha_{R}$ and $\alpha_{\tau}$ can be written as a series in $\alpha_{s} / \pi$ in any given renormalization scheme. In addition we demand that effective charges should also be linear in $\alpha_{s}$, such as $\Delta R=R_{e^{+} e^{-}}-R_{0}$, and it also must track the bare charge in its coupling to the various quark flavors. (Thus $R_{e^{+} e^{-}}^{2}-R_{0}^{2}=$ $\left(R_{e^{+} e^{-}}+R_{0}\right) \Delta R$ is unacceptable since it is not linear in quark flavor 2). Their relevance is due

${ }^{2}$ We thank Prof. M.A. Braun for discussions on this point 
to the fact that they satisfy the renormalization group equation with the same coefficients $\beta_{0}$ and $\beta_{1}$ as $\alpha_{s}$.

As a consequence of the Mean Value Theorem applied to eq.(11), $\alpha_{R}$ and $\alpha_{\tau}$ are simply related by a scale shift $\alpha_{\tau}(M)=\alpha_{R}\left(\sqrt{s^{*}}\right)$. However we can use NLO leading twist QCD to predict

$\lambda_{\tau}=\frac{\sqrt{s^{*}}}{M}=\exp \left[-\frac{19}{24}-\frac{169}{128} \frac{\alpha_{R}(M)}{\pi}+\cdots\right]$,

This result was first obtained in [5] by using the NNLO, and it is a particular example of a "commensurate scale relation" [5 which relate observables at two different scales. We will now see that it is due to the fact that both effective charges evolve with universal $\beta_{0}$ and $\beta_{1}$ coefficients [3].

\section{Tests of PQCD for a general observable}

The previous relations can be generalized to an arbitrary observable $O(\sqrt{s})$, with an associated effective charge $\alpha_{O}$, by defining new effective charges

$\alpha_{f}(M) \equiv \frac{\int_{0}^{M^{2}} \frac{d s}{M^{2}} f\left(\frac{s}{M^{2}}\right) \alpha_{O}(\sqrt{s})}{\int_{0}^{M^{2}} \frac{d s}{M^{2}} f\left(\frac{s}{M^{2}}\right)}$,

where we can choose $f(x)$ to be any smooth, integrable function of $x=s / M^{2}$. Once again

$\alpha_{f}(M)=\alpha_{O}\left(\sqrt{s_{f}^{*}}\right), \quad 0 \leq s_{f}^{*} \leq M^{2}$.

Let us remark that this relation involves only data for $O(\sqrt{s})$ and is therefore a self-consistency test of the leading twist QCD approximation. The relation between the commensurate scales is obtained if we consider the running of $\alpha_{O}$ up to third order

$$
\begin{gathered}
\frac{\alpha_{O}(\sqrt{s})}{\pi}=\frac{\alpha_{O}(M)}{\pi}-\frac{\beta_{0}}{4} \ln \left(\frac{s}{M^{2}}\right)\left(\frac{\alpha_{O}(M)}{\pi}\right)^{2}+ \\
\frac{1}{16}\left[\beta_{0}^{2} \ln ^{2}\left(\frac{s}{M^{2}}\right)-\beta_{1} \ln \left(\frac{s}{M^{2}}\right)\right]\left(\frac{\alpha_{O}(M)}{\pi}\right)^{3},
\end{gathered}
$$

and we substitute for $\alpha_{O}$ in eq. (4), to find

$$
\begin{gathered}
\frac{\alpha_{f}(M)}{\pi}=\frac{\alpha_{O}(M)}{\pi}-\frac{\beta_{0}}{4}\left(\frac{I_{1}}{I_{0}}\right)\left(\frac{\alpha_{0}(M)}{\pi}\right)^{2}+ \\
\frac{1}{16}\left[\beta_{0}^{2}\left(\frac{I_{2}}{I_{0}}\right)-\beta_{1}\left(\frac{I_{1}}{I_{0}}\right)\right]\left(\frac{\alpha_{O}(M)}{\pi}\right)^{3},
\end{gathered}
$$

where $I_{l}=\int_{0}^{1} f(x)(\ln x)^{l} d x$. Thus, $\lambda_{f} \equiv \frac{\sqrt{s_{f}^{*}}}{M}$ is

$\lambda_{f}=\exp \left[\frac{I_{1}}{2 I_{0}}+\frac{\beta_{0}}{8}\left(\left(\frac{I_{1}}{I_{0}}\right)^{2}-\frac{I_{2}}{I_{0}}\right) \frac{\alpha_{O}(M)}{\pi}\right]$.

Since $\lambda_{f}$ is constant to leading order, $\alpha_{f}$ satisfies the same renormalization group equation as $\alpha_{O}$ with the same coefficients $\beta_{0}$ and $\beta_{1}$; i.e., $\alpha_{f}$ is an effective charge.

\subsection{Example: $R_{e^{+} e^{-}}$data.}

For illustrative purposes, we will set $O=$ $R_{e^{+} e^{-}}$, since the data is known to present problems in some energy ranges, but a good agreement with QCD in others. We will see that our test is able to detect these problematic regions. In order to suppress the low energy effects, where nonperturbative effects are important, we shall set $f(x)=x^{k}$, with $k$ some positive number. Thus

$\alpha_{k}(M)=\alpha_{R}\left(\lambda_{k} M\right) \quad$ with $\quad \lambda_{k}=e^{\frac{-1}{2(1+k)}}$,

When comparing with $R_{e^{+} e^{-}}$data, we take into account finite mass effects using [7]:

$$
\begin{aligned}
R_{e^{+} e^{-}}(\sqrt{s}) & =3 \sum_{1}^{f} q_{i}^{2} T\left(v_{i}\right)\left[1+g\left(v_{i}\right) \frac{\alpha_{R}(\sqrt{s})}{\pi}\right] \\
& \equiv R_{0}(\sqrt{s})+R_{S c h}(\sqrt{s}) \frac{\alpha_{R}(\sqrt{s})}{\pi} \\
g(v) & =\frac{4 \pi}{3}\left[\frac{\pi}{2 v}-\frac{3+v}{4}\left(\frac{\pi}{2}-\frac{3}{4 \pi}\right)\right]
\end{aligned}
$$

where $v_{i}$ is the velocity of the initial quarks in their CM frame. The $T\left(v_{i}\right)=v_{i}\left(3-v_{i}^{2}\right) / 2$ factor is the parton model mass dependence and $g(v)$ is a QCD modification of the Schwinger correction. The quark masses have been taken as effective parameters which provide a good fit to the smeared data. These corrections spoil eq.(6), but they are only important near the quark thresholds. Since we recover eq.(2) at higher energies, our study is restricted to this regime. Since the data is on annihilation into hadrons, not quarks, we cannot use directly our formulae, although we can define smeared quantities [7] as follows:

$\bar{R}(\sqrt{s})=\frac{\Delta}{\pi} \int_{0}^{\infty} \frac{R\left(\sqrt{s^{\prime}}\right)}{\left(s-s^{\prime}\right)^{2}-\Delta^{2}} d s^{\prime}$ 

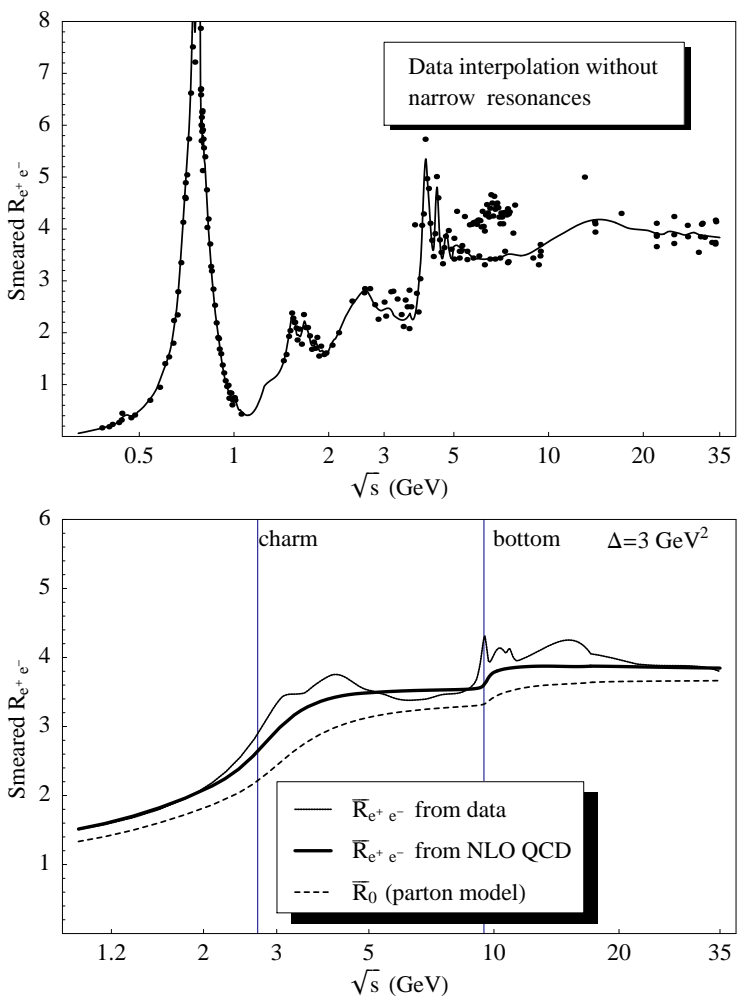

Figure 1.a) Interpolation of the central values of $R_{e^{+} e^{-}}$ data (see 3. for references). Note the discrepancy in the central values of experiments between 5 and $10 \mathrm{GeV}$. b) Smeared $R_{e^{+} e^{-}}$.

The smearing of $R_{e^{+} e^{-}}$over a range of energy, $\Delta E$, is indeed a smearing over time $\Delta t=1 / \Delta E$, where an analysis in terms of quarks and gluons is appropriate. We have used the standard value $\Delta=3 \mathrm{GeV}^{2}[7,8]$. The smearing effect can be seen by comparing the data interpolation in Fig.1.a, (see [3] for references) with Fig. 1.b. Note that any fit using the QCD functional dependence will always satisfy eq.(6). That is why we have parameterized the narrow resonances with their Breit-Wigner form, and we have interpolated the remaining data (see [3] for details).

Finally, using eqs.(7) and (9), we define smeared charges:

$\bar{\alpha}_{R}(\sqrt{s})=\frac{\bar{R}_{e^{+} e^{-}}(\sqrt{s})-\bar{R}_{0}(\sqrt{s})}{\bar{R}_{S c h}(\sqrt{s})}$, and similarly for $\bar{\alpha}_{k}$. As we have just commented, the smeared charges are expected to satisfy eq. (5) at energies where the threshold corrections are negligible.

In Fig.2.a we show the comparison between $\bar{\alpha}_{R}\left(\sqrt{s^{*}}\right)$ and $\bar{\alpha}_{k}\left(\sqrt{s^{*}} / \lambda_{k}\right)$. The rather poor agreement for $\alpha_{0}$ is due to the fact that the low energy region is not suppressed enough. In contrast there is a fairly good agreement for $\alpha_{1}$ in several regions, which disappears when the scales are not shifted as in eq.(6). As we have commented there are two regions of particular interest: First, from 5 to $10 \mathrm{GeV}$, since the data from two different experiments are incompatible (see Fig.1.a and ref.[9]). Even though we have kept the most recent data in our calculations, their central values are systematically lower than the QCD predictions. Our test correctly points out this incompatibility.

Second, we show in Fig. 2.b. the energy region of the real $\tau$. taking into account that we are just using LO QCD and central data values, the agreement is fairly good, which supports the applicability of PQCD at energies near the physical $\tau$ lepton. Nevertheless, at $\sqrt{s} \sim 1.5 \mathrm{GeV}$, it seems that the there is an small discrepancy of the order of $6-7 \%$, which also supports previous claims that $R_{e^{+} e^{-}}$data is lower than expected from $R_{\tau}$ data 10 .

Note, however, that we have reached our conclusions using only data on $R_{e^{+} e^{-}}$.

\section{Measuring the QCD Gell-Mann-Low $\Psi$ function.}

Given an observable $O(\sqrt{s})$, let us generalize the definition of new effective charges to include arbitrary scales in the integral

$O_{k}(\Lambda) \equiv C \int_{\Lambda_{1}^{2}}^{\Lambda_{2}^{2}} \frac{d s}{\Lambda^{2}}\left(\frac{s}{\Lambda^{2}}\right)^{k} O(\Lambda)$.

The generalization of eq. (4) to obtain an effective charge $\alpha_{k}$ is straightforward. Again we find a commensurate scale relation $\alpha_{k}(\Lambda)=\alpha_{O}\left(\Lambda_{k}\right)$, with $\Lambda_{k}$ predicted by QCD 惯]. But if we differentiate

$\frac{d O_{k}(\Lambda)}{d \log \Lambda}=$ 



Figure 2.a) Comparison between $\bar{\alpha}_{R}\left(\sqrt{s^{*}}\right)$ and different $\bar{\alpha}_{k}$ moments at $M=\sqrt{s^{*}} / \lambda_{k}$. The dotted line shows how the agreement is spoilt if we do not shift $\sqrt{s^{*}}$ to $M$. b) Comparison between $\bar{\alpha}_{R}\left(\sqrt{s^{*}}\right)$ and different $\bar{\alpha}_{k}$ moments at $M=\sqrt{s^{*}} / \lambda_{k}$ in the low energy region.

$$
2 C\left[\lambda_{2}^{2 k+2} O\left(\Lambda_{2}\right)-\lambda_{1}^{2 k+2} O\left(\Lambda_{1}\right)\right]-(2 k+2) O_{k}(\Lambda)
$$

which is basically $\beta_{Q C D}$. Note that we have defined $\lambda_{i}=\Lambda_{i} / \Lambda$. Indeed what we have obtained is the Gell-Mann-Low QCD function $\Psi$ (see ref.|1]), which finally can be written in terms of the $o b$ servable at three energies

$$
\begin{aligned}
\beta_{Q C D}(\Lambda) & \simeq \Psi(\Lambda)= \\
& \frac{k+1}{O^{0}}\left[\frac{\lambda_{2}^{2 k+2} O\left(\Lambda_{2}\right)-O(\Lambda)}{\lambda_{2}^{2 k+2}-1}-O\left(\Lambda_{k}\right)\right] .
\end{aligned}
$$

The advantages of this method are that it eliminates the errors from finite difference approximations, and that it is competitive with a standard fit but it has very different systematics.

\section{Summary}

The relation between $R_{e^{+} e^{-}}$and $R_{\tau}$, as well as the ideas of commensurate scale relations, have motivated a number of new tests of perturbative QCD, as well the means for measuring the QCD $\Psi$ Gell-Mann-Low function. The advantage of these methods is that, although they are inspired in a relation between two observables, at the end they only relate one observable with itself.

These tests, which are renormalization scheme and scale independent, are applicable over wide energy ranges to any observable which defines an effective charge. As an example, we have tested the self-consistency of existing $R_{e^{+} e^{-}}$data according to PQCD, finding a good agreement in the real $\tau$ region but incompatibilities around the $1.5 \mathrm{GeV}$ region and in the range of 5 to $10 \mathrm{GeV}$, supporting previous claims in the literature.

The method to measure the Gell-Mann-Low function eliminates the errors present in finite difference techniques, and it is competitive with standard fits although with different systematics.

\section{REFERENCES}

1. E. Braaten, Phys. Rev. Lett. 60 (1988), Phys. Rev. D39 1458 (1989); E. Braaten, S.Narison and A.Pich, Nucl. Phys. B373 581 (1992).

2. F. Le Diberder and A.Pich, Phys. Lett. B289 165(1992); S. Narison and A. Pich, Phys. Lett. B304 359 (1993), S.Narison, Phys. Lett. B358 113 (1995) and Phys. Lett. B361 121 (1995), M. Girone and M. Neubert, Phys. Rev. Lett. 763061 (1996); S. Groote, J. G. Korner and A. A. Pivovarov. Phys. Lett. B407 66 (1997); M. Davier, hep-ph/9802447; R.Barate et al. Eur. Phvs. J. C4 409 (1998). K.Ackerstaff et al., hep-ex/9808019.

3. S. J. Brodsky, J. R. Peláez and N. Toumbas, Phys. Rev. D60 037501 (1999).

4. S. J. Brodsky, C. Merino and J. R. Peláez. hepph/9906382. slac-pub-8033. To appear in Phys.Rev.D.

5. S.J.Brodsky and H.J.Lu Phys. Rev. D51 3652(1995). S. J. Brodsky and J. Rathsman. hep-ph/996339.

6. G. Grunberg, Phys. Rev. D29 2315 (1984).

7. E. C. Poggio, H. R. Quinn and S. Weinberg, Phys. Rev. D13 1958 (1976). T. W. Appelquist and H. D. Politzer, Phys. Rev. Lett. 34, 43 (1975); Phys. Rev. D12 1404 (1975). J. Schwinger, Particles, Sources and Fields, Vol.II, Addison-Wesley, New York, 1973.

8. A. C. Mattingly and P.M. Stevenson, Phys. Rev. D49 437 (1994).

9. M. L. Swartz, Phys. Rev. D53 5268 (1996).

10. S. Groote et al., Phys. Rev. Lett. 792763 (1997). 